\title{
Diabulimia: an easily missed diagnosis?
}

\author{
CARL DAVID LEITH VAN HEYNINGEN, ${ }^{1}$ KARTHIKEYINI SUJAY MANOHARAN ${ }^{2}$
}

\section{Introduction}

Diabulimia is an eating disorder where the patient voluntarily omits insulin to achieve weight loss. This case report aims to increase awareness of diabulimia as a patient factor in recurrent diabetic ketoacidosis (DKA). A high index of clinical suspicion with appropriate screening in patients with type 1 diabetes is required to better identify children with comorbid eating disorders. ${ }^{1-3}$ The subsequent management may be far from straightforward and requires realistic goals with the initial focus on establishing glycaemic stability. ${ }^{4}$

Key words: recurrent diabetic ketoacidosis, type 1 diabetes, adolescent, eating disorder, diabulimia

\section{Case report}

A 14-year-old girl was brought to hospital by ambulance following referral by primary care. She had been complaining of headaches, dizziness and two months of insatiable thirst and polyuria. She had lost nearly $22 \mathrm{~kg}$ in weight and her initial blood glucose was $21.2 \mathrm{mmol} / \mathrm{L}$. A diagnosis of new onset type 1 diabetes was made. After her acute management she was commenced on a basal bolus regimen of 0.5 units $/ \mathrm{kg} /$ day inclusive of fixed doses NovoRapid (5, 5 and 6 units) with 14 units Lantus daily.

During the initial diabetic consultation, self-harm marks were identified on both her arms. When asked about these marks she did not want to talk. Her father openly disclosed that she had selfharmed at school following an episode of bullying. Her mother and father had recently divorced. She admitted to missing her mother as she lived with her father. She also lived with her sister and at weekends saw her grandparents.

Following completed diabetic education, she was discharged home with outpatient follow-up arranged. In the subsequent weeks she continued to experience high blood sugars. Her $\mathrm{HbA}_{1 \mathrm{c}}$ remained at $118 \mathrm{mmol} / \mathrm{L}$. Her Lantus and correction doses were increased. In view of further self-harm marks and new concerns

Paediatric Emergency Medicine Educational Fellow, Leicester Royal Infirmary, UK

2 Higher Speciality Trainee, Paediatrics, Norfolk and Norwich University Hospitals NHS Foundation Trust, UK

Address for correspondence: Dr Carl David Leith van Heyningen Paediatric Emergency Department, Leicester Royal Infirmary, Infirmary Square, Leicester LE1 5WW, UK

E-mail: carlvanheyningen@aol.com

Br J Diabetes 2018;18:167-170

https://doi.org/10.15277/bjd.2018.196 of weight loss, she was urgently referred for psychological assessment and support. In the meantime, her glycaemic control worsened and she was soon admitted to hospital (three months post-diagnosis) with DKA. She admitted to not taking her insulin having become frustrated with her injections. She was not alarmed by being in hospital and remained unmotivated to improve her self-care. In addition to omitting insulin, she used inappropriate injection sites and ate meals erratically, often skipping breakfast.

This behaviour continued for months. At her follow-up appointment with the psychologist she was found to have multiple bruises. In addition to bullying at school, she had been enduring cyber-bullying on social media. She often binge ate in response to her emotional distress. An urgent referral was then made to community adolescent mental health services (CAMHS). Sadly, she never attended this appointment despite frequent rescheduling efforts.

Over the next year she had three further admissions with DKA. On two occasions her DKA was life threatening. She spent a total of four days in intensive care. Whilst recovering in intensive care she had volunteered "Once I go home, I will not inject myself. I will become unwell which is the best way to get back into a coma. This is what I want." Concerns were escalated. She was started on a longer acting insulin (Tresiba) and she was urgently rereferred to CAMHS and an urgent multidisciplinary team (MDT) meeting was scheduled. In spite of these plans, she was soon readmitted a fifth time with DKA, again following insulin omission.

On this occasion she was deemed unfit for hospital discharge. Inpatient psychiatric assessment was sought. Depression was suspected. Her initial psychiatric assessment with the crisis team revealed severe body image anxiety, fears of weight gain and low self-esteem. On the Eating Disorder Examination Questionnaire she scored high. She also expressed suicidal ideation and admitted to low mood and tearfulness. She described her desire for a mother figure and expressed grief regarding her parents' separation.

An MDT meeting was held. Individual therapy was prioritized to address her personal difficulties. Family therapy was arranged to consider family dynamics and the need for setting boundaries. Further psychiatric input was planned to further explore her suicidal ideation. Her diabetic education was revised, aiming to empower her self-care with regard to self-injection, carbohydrate counting and correction dosing with her insulin.

It has taken over a year after diagnosis of her diabetes before her concordance began to improve. She had gained improved glycaemic control ( $\left.\mathrm{HbA}_{1 \mathrm{c}} 76 \mathrm{mmol} / \mathrm{L}\right)$, improved hypoglycaemic 
awareness alongside improved self-care. Whilst obstacles persisted (including an unhealthy diet, infrequent exercise and continued low self-esteem), she had no further life-threatening hospital admissions and continued to show increased resilience with ongoing mental health support.

\section{Discussion}

'Diabulimia' is difficult to define. For some it is a broad term describing any deliberate insulin omission with various underlying motives and psychiatric aetiologies. For others it is a specific term referring to an eating disorder affecting patients with type 1 diabetes where the primary goal is weight loss. Its recognition is equally challenging, and whilst we continue to debate this term it is vitally important we don't ignore this very real problem.

High rates of disordered eating, body image distortion and intentional insulin misuse for weight loss among young people with diabetes are increasingly recognized. 5,6 In comparison to their peers (adolescents without type 1 diabetes), these children have a high prevalence of disordered eating. ${ }^{7}$ Whilst these issues are well known to affect adolescent girls, ${ }^{8}$ affected boys must not be forgotten. ${ }^{9}$

As in our example, consequences include higher $\mathrm{HbA}_{1 \mathrm{c}}$ values, increased risk of short-term ketoacidosis and longer-term higher rates of retinopathy, nephropathy, vascular disorders and premature death. ${ }^{8}$ This particular teenage girl came dangerously close to death.

The aetiology of our patient's recurrent DKA is complex. Whilst a variety of medical, social, economic and family factors (see Box 1) are at play, ${ }^{10}$ the prevention of further episodes requires recognition that the common causal link is inadequate insulin. In this case the cause was repeated intentional underdosing and omission of insulin. Calorie wasting through hyperglycaemia and glycosuria appears to be a likely motive. In such cases of recurrent DKA, insulin omission is a leading cause. ${ }^{1}$ The Diagnostic and Statistical Manual of Mental Disorders, 4th Edition (DSM IV) currently classifies such a diagnosis as 'misuse of other medications as a symptom of bulimia nervosa'. Previously this may also have been referred to as 'eating disorder not otherwise specified'. Regardless of the label, as already suggested, diabulimia clearly represents a serious and dangerous emerging problem. After one hospitalisation for DKA, patients have a 10\% greater risk of dying within 5 years. ${ }^{11}$ After four hospitalizations this rises to a $30 \%$ increased risk of dying within 6 years. ${ }^{11}$ Prevention is paramount. Early identification of underlying mental ill health is essential.

Detection requires a high index of clinical suspicion. ${ }^{2,3}$ In addition to risk factors for eating disorders in the general population (Box 2), people with diabetes are arguably at higher risk. Candler et al discuss why this might be. ${ }^{12}$ Young newly diagnosed people with diabetes are vulnerable. They are subjected to regular weight checks, carbohydrate counting and painful injections. Their entire lifestyle is in constant flux. Their parents are no doubt anxious and, for all concerned, acceptance of their condition is difficult. Emotional turmoil is to be expected which, alongside the increased focus on diet and weight, can under-
Box 1. Influences on adherence to diabetic treatment in adolescence

- Social relationships

- Peer pressure

- Increasing independence from family

- Increasing time spent away from family

- Pubertal development (both physical and behavioural)

- Psychological sequelae of type 1 diabetes

- Co-morbidity with eating disorders

Box 2. Risk factors for eating disorders in the general population

- Female sex

- Weight gain and being overweight

- Dietary restraint and dieting

- Early puberty compared with peers

- Low self-esteem

- Disturbed family functioning, disturbed parental eating attitudes

- Peer and cultural influences

- Personality traits (perfectionism, childhood anxiety disorder diagnosed before age of 8 years).

standably seed the start of many eating disorders. Such struggles may remain hidden, with shame and embarrassment being obstacles to early recognition. ${ }^{12,13}$

Health professionals must be vigilant when children present with unexplained elevations in $\mathrm{HbA}_{1 \mathrm{c}}$ values, repeated admissions with DKA, physical symptoms of hair loss or thinning, dizziness, abdominal pain or headache. Behavioural traits such as not eating with family, frequently not having their insulin available at meal times, great interest in body shape and size or overexertion can be clues. Clearly weight loss and amenorrhoea would be red flags in a pubescent female.

Diabetes educators, psychologists or clinicians may be the first people to uncover such details which may be initially overlooked. Further warning signs might include poor school attendance, neglect of general diabetic management, erratic clinic attendances, preoccupation with meal planning and food composition, depressive symptoms including low mood, fatigue, disrupted sleep and poor appetite (to be noted that poorly controlled diabetes can in itself directly contribute to depressive symptoms). Screening tools exist, including the Eating Attitudes Test ${ }^{14}$ and the modified Diagnostic Survey for Eating Disorders tool, 15 both of which are validated for use in diabetes. Others, including the Eating Disorder Examination Questionnaire, appear not to identify the aforementioned increased prevalence of eating disorders among people with diabetes. ${ }^{16}$ It is, of course, important to recognise that a negative screening result may simply reflect a teenager too ashamed to disclose issues, hence the importance of de-stigmatising language alongside the normalisation of discussing mental health. 
Once diagnosis is suspected it should be confirmed with clinical interview. The diagnosis may initially be disclosed within the diabetes team and then prompt further referral to psychology and/or community mental health services.

Once a diagnosis or formulation is reached, outpatient follow-up should include shared care between eating disorder and diabetes teams. The initial focus should be on glycaemic stability (to avoid further life-threatening DKA episodes), then on the specific eating disorder symptoms (to address underlying issues). Finally, optimal diabetic management (to prevent future complications) should be addressed. One of the most important aspects of treatment is the establishment of a positive rapport. Patients need to learn to trust their team. They must become comfortable communicating honestly despite frequent feelings of shame and low mood. Through avoiding judgement and promoting acceptance, patients are more likely to engage in ongoing care plans.

Within the context of recurrent insulin restriction and severe hyperglycaemia, the initial treatment goal should be the avoidance of DKA. At a minimum, establish a commitment to routinely taking basal insulin doses. Incremental goals can then be set. As patients' symptoms improve, less frequent follow-up will be necessary. Weight checks can trigger anxiety and hence should routinely be blinded to the subject. Weight stability is often more important than weight loss and pre-occupation with weight is to be avoided. Physical examination should focus on nutritional state and include assessment of injection sites. Insulin devices and technique should also be periodically checked during clinic visits. ${ }^{17}$

Innovative use of diabetic medications (for example, transition to split-mixed insulin or pre-mixed insulin) may improve adherence until patients are ready for more self-led plans. Pump therapy is also showing promise to improving glycaemic control in these difficult to manage patients. ${ }^{18}$ Discussion around nutrition must also be carefully handled. The 'total diet' approach focuses on 'moderation and proportionality' in the context of a healthy lifestyle. ${ }^{19}$

Realistic blood glucose goals should be established gradually. This ensures continued positive motivation and reduced rates of burnout with reduced risks of relapse or treatment drop-out. As seen here, a comprehensive treatment plan for co-morbid mental health (eating disorder and depression) is also essential. It should ideally involve weekly contact with a mental health professional, although this care could be shared with the community diabetes team. Frequent communication between MDT members with active patient support can establish sustainable improvements, preventing the worrisome pattern of recurrent DKA admissions we all dread.

\section{Conclusion}

Adolescents with type 1 diabetes mellitus are at high risk of co-morbid eating behaviours. Insulin omission is a particular concern as it can lead to recurrent DKA and associated mortality and morbidity. At this developmental stage, behaviours around weight management and insulin misuse are particularly prevalent. Often a holistic approach to care is required. This includes

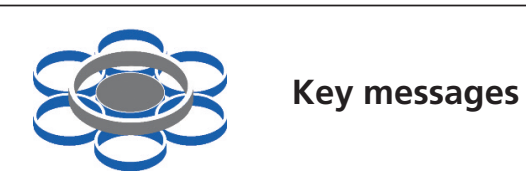

- Maintain a high index of suspicion for the presence of an eating disturbance

- Consider in cases of unexplained raised $\mathrm{HbA}_{1 \mathrm{c}}$ and recurrent diabetic ketoacidosis

- Early detection and referral is important; consider the use of a screening tool

- A multidisciplinary supportive and non-stigmatising approach is required

- Management goals may need to be adjusted, with medical stability being the first priority

broadening the MDT to include, for example, eating disorder professionals, psychologists and frequently psychiatrists. ${ }^{20}$

With so much at stake (not including the effects upon families, professionals and healthcare systems), diabulimia awareness has never been more important. Young people deserve timely and appropriate intervention in cases of co-morbid mental health, eating disorder and other conditions (for example, depression) to protect them against the spiralling dangers of insulin omission and recurrent DKA.

\section{Conflict of interest None \\ Funding None}

\section{References}

1. Golden MP, Herrold AJ, Orr DP. An approach to prevention of recurrent diabetic ketoacidosis in the paediatric population. J Pediatr 1985;107: 195-200.

2. Criego A, Crow S, Goebel-Fabbri AE, Kendal D, Parkin C. Eating disorders and diabetes: screening and detection. Diabetes Spectrum 2009; 22:143-6

3. Urbanski P, Powers M, Taylor D. The diabetes educator's role in managing eating disorders and diabetes. Diabetes Spectrum 2009;22:159-62.

4. Goebel-Fabbri AE, Uplinger N, Gerken S, Mangham D, Criego A, Arkin C. Outpatient management of eating disorders in type 1 diabetes. Diabetes Spectrum 2009;22:147-52.

5. Bryden KS, Neil A, Mayou RA, et al. Eating habits, body weight, and insulin misuse. A longitudinal study of teenagers and young adults with type 1 diabetes. Diabetes Care 1999;22:1956-60.

6. Bächle C, Stahl-Pehe A, Rosenbauer J. Disordered eating and insulin restriction in youths receiving intensified insulin treatment: results from a nationwide population-based study. Int J Eat Disord 2016;49:191-6. https://doi.org/10.1002/eat.22463

7. Young $V$, Eiser $C$, Johnson $B$, et al. Eating problems in adolescents with type 1 diabetes: a systematic review with meta-analysis. Diabet Med 2013;30:189-98. https://doi.org/10.1111/j.1464-5491.2012.03771.x

8. Neumark-Sztainer D, Patterson J, Mellin A, et al. Weight control practices and disordered eating behaviors among adolescent females and males with type 1 diabetes: associations with sociodemographics, weight concerns, familial factors, and metabolic outcomes. Diabetes Care 2002; 25:1289-96.

9. Hepworth K. Eating disorders today, not just a girl thing. J Christian Nurs 2010;27:236-41; quiz 242-3.

10. Goebel-Fabbri AE, Fikkan J, Franko DL, Pearson K, Anderson BJ, Weinger K. Insulin restriction and associated morbidity and mortality in women with type 1 diabetes. Diabetes Care 2008;31:415-19. https://doi.org/10.2337/dc07-2026 


\section{CASE REPORT}

11. Gibb FW, Teoh WL, Graham J, Lockman A. Previous diabetic ketoacidosis is associated with a significant risk of death in the following 5 years. American Diabetes Association 2015, Boston, Massachusetts, Scientific Sessions.

12. Candler T, Murphy R, Pigott A, Gregory JW. Fifteen-minute consultation: diabulimia and disordered eating in childhood diabetes. Arch Dis Child (Ed Practice) 2018;103:118-23. http://dx.doi.org/10.1136/archdischild2017-312689

13. Davidson J. Diabulimia: how eating disorders can affect adolescents with diabetes. Nurs Stand 2013;29:44-9. https://doi.org/10.7748/ns.29.2.44.e7877

14. Garner DM, Olmsted MP, Bohr Y, Garfinkel PE. The Eating Attitudes Test: psychometric features and clinical correlates. Psychol Med 1982;12:871-8.

15. O'Connell D. Assessment. In: Dual Disorders: Essentials for Assessment and Treatment. New York: Hawthorne Press, 1998: 17-30.

16. Keane S, Clarke M, Murphy M, et al. Disordered eating behaviour in young adults with type 1 diabetes mellitus. J Eating Disord 2018;6:9 https://doi.org/10.1186/s40337-018-0194-2

17. Muralidharan S, Datta V, Palanivel V. Recurrent diabetic ketoacidosis in an adolescent. Arch Dis Child 2009:94:365. https://doi.org/10.1136/adc.2008.146803

18. Pinhas-Hamiel O, Graph-Barel C, Boyko V, et al. Long-term insulin pump treatment in girls with type 1 diabetes and eating disorders--is it feasible? Diabetes Technol Ther 2010;12:873-8. https://doi.org/10.1089/dia.2010.0049

19. Nitzke S, Freeland-Graves J. Position of the American Dietetic Association: total diet approach to communicating food and nutrition information. J Am Diet Assoc 2007;107:1224-32.

20. Larrañaga A, Docet MF, García-Mayor RV. Disordered eating behaviors in type 1 diabetic patients. World J Diabetes 2011;2:189-95. https://doi.org/10.4239/wjd.v2.i11.189 